\title{
Diagnosing COVID-19 pneumonia from x-ray and CT images using deep learning and transfer learning algorithms
}

Maghdid, Halgurd, Asaad, Aras, Ghafoor, Kayhan Zrar, Sadiq, Ali Safaa, Mirjalili, Seyedali, et al.

Halgurd S. Maghdid, Aras T. Asaad, Kayhan Zrar Ghafoor, Ali Safaa Sadiq, Seyedali Mirjalili, Muhammad Khurram Khan, "Diagnosing COVID-19 pneumonia from $x$-ray and CT images using deep learning and transfer learning algorithms," Proc. SPIE 11734, Multimodal Image Exploitation and Learning 2021, 117340E (12 April 2021); doi: 10.1117/12.2588672 


\title{
Diagnosing COVID-19 Pneumonia from X-Ray and CT Images using Deep Learning and Transfer Learning Algorithms
}

\author{
Halgurd S. Maghdid ${ }^{a^{*}}$, Aras T. Asaad ${ }^{\text {b }}$, Kayhan Zrar Ghafoor ${ }^{c}$, Ali Safaa Sadiq ${ }^{\text {d }}$, \\ Seyedali Mirjalili ${ }^{\text {e }}$, Muhammad Khurram Khan ${ }^{\mathrm{f}}$ \\ $\mathrm{a}^{*}$ Halgurd S. Maghdid is with the Department of Software Engineering, Faculty of Engineering, Koya \\ University, Kurdistan Region-F.R.Iraq. email: halgurd.maghdid@koyauniversity.org. \\ ${ }^{\mathrm{b}}$ Aras Asaad is with the Oxford Drug Design, Oxford Centre for Innovation, Oxford, OX1 1BY, UK. \\ email: aras.asaad@oxforddrugdesign.com \\ ${ }^{c}$ Department of Computer Science, Knowledge University, University Park, Kirkuk Road, 44001 \\ Erbil, Iraq. Email: kayhan.zrar@knu.edu.iq. \\ d Ali Safaa Sadiq is with the School of Mathematics and Computer Science, University of \\ Wolverhampton, Wulfruna Street, Wolverhampton, WV1 1LY, UK. email: Ali.Sadiq@wlv.ac.uk. \\ ${ }^{\mathrm{e}}$ Seyedali Mirjalili is with the Centre for Artificial Intelligence Research and Optimisation, Torrens \\ University Australia, Fortitude Valley, Brisbane, 4006 QLD, Australia. email: ali.mirjalili@gmail.com. \\ ${ }^{\mathrm{f}}$ Muhammad Khurram Khan is with the Centre of Excellence in Information Assurance, King Saud \\ University, Riyadh, Saudi Arabia. email: mkhurram@KSU.EDU.SA
}

\begin{abstract}
The novel coronavirus 2019 (COVID-19) first appeared in Wuhan province of China and spread quickly around the globe and became a pandemic. The gold standard for confirming COVID-19 infection is through Reverse Transcription-Polymerase Chain Reaction (RT-PCR) assay. The lack of sufficient RT-PCR testing capacity, false negative results of RT-PCR, time to get back the results and other logistical constraints enabled the epidemic to continue to spread albeit interventions like regional or complete country lockdowns. Therefore, chest radiographs such as CT and X-ray can be used to supplement PCR in combating the virus from spreading. In this work, we focus on proposing a deep learning tool that can be used by radiologists or healthcare professionals to diagnose COVID-19 cases in a quick and accurate manner. However, the lack of a publicly available dataset of X-ray and CT images makes the design of such AI tools a challenging task. To this end, this study aims to build a comprehensive dataset of X-rays and CT scan images from multiple sources as well as provides a simple but an effective COVID-19 detection technique using deep learning and transfer learning algorithms. In this vein, a simple convolution neural network $(\mathrm{CNN})$ and modified pre-trained AlexNet model are applied on the prepared X-rays and CT scan images. The result of the experiments shows that the utilized models can provide accuracy up to $98 \%$ via pre-trained network and $94.1 \%$ accuracy by using the modified CNN.
\end{abstract}

Keywords: COVID-19, deep learning, coronavirus detection, CT scan image, CNN.

\section{INTRODUCTION}

The novel COVID-19 was first reported in Wuhan city, Hubei Province of China, China in November 2019. A month later, the World Health Organization (WHO) announced that the virus can cause a respiratory disease with clinical presentation of cough, fever and lung inflammation. Although the COVID-19 emerged in China, it has now been identified in many other countries around the globe [1], [2]. On January 30, 2020, WHO announced this pandemic as public health emergency. This is not only

Multimodal Image Exploitation and Learning 2021, edited by Sos S. Agaian, Vijayan K. Asari,

Stephen P. DelMarco, Sabah A. Jassim, Proc. of SPIE Vol. 11734, 117340E

(c) 2021 SPIE · CCC code: $0277-786 X / 21 / \$ 21 \cdot$ doi: 10.1117/12.2588672 
due to rapid spread of the virus but also because of high rate of mortality. Why this virus has brought the world to a sneeze and also discusses the implications of infectious diseases in our daily life.

Research evidences reports that the coronavirus virus emerged from an animal source, and then sustained human to human transmission. Epicenter in Wuhan, China is a ground zero for the COVIDThe world is so concerned about COVID-19 to which no one has natural immunity. This is the main reason that the number of positive COVID-19 cases are growing significantly [3]. Since the first report of the virus till March 30, 2020, there were over 750,000 cases and 36000 deads worldwide. That's why, WHO announced that the novel COVID-19 outbreak as a pandemic [4]. Recently, Italy, United States and Spain overtakes the China in number of fatalities.

The symptoms of coronavirus is complicated and could appear as fever, cough and severe headache. One of the techniques to diagnose COVID-19 is the Nucleic Acid Test (NAT) scan. The NAT is used to identify a specific nucleic acid sequence and species of organism, mainly a virus or bacteria that causes disease in blood, tissue or urine. Although NAT is important in diagnosing COVID-19, Computed Tomography (CT) scans considered as an accurate way for detecting the severity of infected lung likely to be associated with COVID-19 [5]. As recommended by National Health Commission of China, radiographic presentation of pneumonia is required for clinical diagnostic standard in Hubei Province [6], which stresses on the importance of CT scan images for the detection of COVID-19 lung inflammation severity.

Recently, a surge of COVID-19 patients has introduced long queues at hospitals for CT scan im- age examination. This leads to a serious risk of cross-infection with other patients and consequently overloads the medical system. Moreover, the number of radiologists is relatively far smaller than the number of patients which can lead to late detection and quarantine of infected people and less efficient treatment of patients [6]. As a result, for instance, recently in Italy, hospitals have had to give priority to people with a significant fever and shortness of breath over others with less severe symptoms [7]. The rapid spread of COVID-19 and the overwhelming demand for diagnosis has driven researchers to develop more intelligent, highly responsive and efficient diagnosis methods. One diagnosis method, handled by the radiologists, is the manual lung infection quantification. Further, there is an AI-based automated pneumonia diagnosis used to identify the density and volume of lesions and opacities of confirmed COVID-19 cases. Such algorithms are able to analyze the results of CT scan images in a short time in comparison to other existing methods [8].

Radiologists use chest CT scan images to follow up the confirmed cases ranging from early to critical stages [8]. The quick progression of lung infection requires multiple CT scan images Interpreting and analyzing these images can be a time consuming and daunting task, especially when manually quantifying the infected regions on the CT scan and X-ray images. Thus, there is a pressing need to develop an intelligent algorithm to accurately and automatically detect COVID-19 cases. Furthermore, it is required to build a complete and ready-to-experiment dataset for research community.

This research first reviews the state-of-the-art solutions to combat COVID-19. Then, we build a preprocessed and comprehensive dataset on X-rays and CT scan images from multiple sources, and provide an accurate COVID-19 detection algorithm using deep learning and transfer learning tools. Further, a modified CNN and AlexNet model as a pre-trained network are applied on the prepared Xrays and CT scan images datasets. After extensive experiments on both datasets, it is shown that the proposed model predicts COVID-19 diagnosis with high accuracy and low response time. To the best of our knowledge, there is no work in the open literature that uses both pretrained and CNN on both CT scan and X-ray images in identifying COVID-19.

From a technical standpoint, we summarise the most important contributions of this paper as follows:

1) We build a ready-to-use dataset of CT scan and X-ray images. For this purpose, we use multiple sources of those images in order to make the proposed model more realistic in detecting COVID-19 cases.

2) We propose a modified CNN model to accurately diagnose COVID-19 and hence delay the fast spread of the virus.

3) In order to accelerate and elevate the accuracy of diagnosis, we also propose a modified pre- 
trained deep learning model to detect COVID-19.

The rest of this paper is organized as follows. Section II provide the literature review on recent advances of developed AI systems for COVID-19 detection. This is followed by presenting an overview of the proposed approach and details of the designed algorithm in section III. Section IV presents the experiments and datasets which are used in the paper. Finally, Section IV concludes the paper.

\section{BACKGROUND}

As it was stated earlier that Computed tomography (CT) scan method is the ideal way for diagnosing novel coronavirus (COVID-19) pneumonia. The authors in [1] have conducted a research intended to build a diagnose system based on deep learning for identifying COVID-19 pneumonia. Their system works with high-resolution CT scan images in diagnosing COVID19, which supports radiologists with their work and helps to control the epidemic. It was interesting that they could manage to collect 46,096 anonymous images from 106 admitted patients, involving 51 cases of laboratory confirmed COVID-19 pneumonia. Besides, they have collected 55 control patients of other diseases admitted in Renmin Hospital of Wuhan University (Wuhan, Hubei province, China). All these CT scan images were prospectively collected to develop and train their proposed model as well as to evaluate and contrast the efficiency of radiologists versus COVID19 pneumonia with the performance of their model.

It is important to mention that their proposed deep learning model exhibits an equivalent performance compared with expert radiologist. Moreover, it could also significantly improve the efficiency of radiologists while doing their clinical practice; which is crucially needed with such outbreaks situations when cases exponentially increasing. Hence, we can highlight here an argument, having such diagnosing system grips numerous potential to alleviate the burden of frontier radiologists in addition to advance the timely COVID19 diagnosis. Furthermore, obtaining an early detection of such highly contagious pandemic will further help in isolation and cure plans, and eventually assists countries in controlling and ending such epidemic [1].

There was also a very important claim highlighted in [1] on the importance of CT scan images in diagnoses the cases of COVID19, which can be considered a much quicker method in comparison with the conventional way using the nucleic acid detection. In additional to its efficiency in diagnosing the infection, it can estimate the level of severity of pneumonia [9]. It was reported that CT results were positive with all 140 laboratory-confirmed COVID-19 patients. Some of these positive cases, CT scan was able to recognize them even within their early stage, which reflects its effectiveness [10], [11]. On the other hand, the National Health and Health Commission of China has reported in their fifth version of COVID-19 diagnostic manual, the radiographic characteristics of pneumonia is integrated the clinical diagnostic standard in Hubei Province.

From the aforementioned discussion, the researchers in [1] were mainly focusing to achieve a model that could closely diagnosis COVID19 to the way radiologists do, but with shorter time. They could achieve a comparable performance to that of expert radiologist with $65 \%$ lesser time taken in diagnosing cases compared to in-clinic radiologist's time. Through, there are still points of improvements available for further enhancing their proposed model and the entire system to be personally accessible by users. This will enable patients as well as suspected cases to have their selfcheck system so they save more time and avoid direct contact that may lead for disseminating the virus to the specialists or nurses.

On the other hand, the authors in [8] have stated that till the time of writing their report, there is no such automatic toolkit to clinically quantify the level of COVID-19 infection for patients. For this motive, they have introduced a deep learning (DL)-based system to automatically segmenting and quantifying the infection spots of the COVID19 patients in addition to obtaining the entire view of lung to be extracted from chest CT scans. To perform image segmentation, a DL-based network named VB- Net was proposed. Authors in [8] have developed a modified 3-D convolutional neural network, which associations V-Net [12] along with the bottleneck structure presented in [13]. The adopted VB- 
Net is presented in Figure 1, which entails of two routes, the dashed boxes highlighting the utilized bottleneck elements within their V-shaped network.
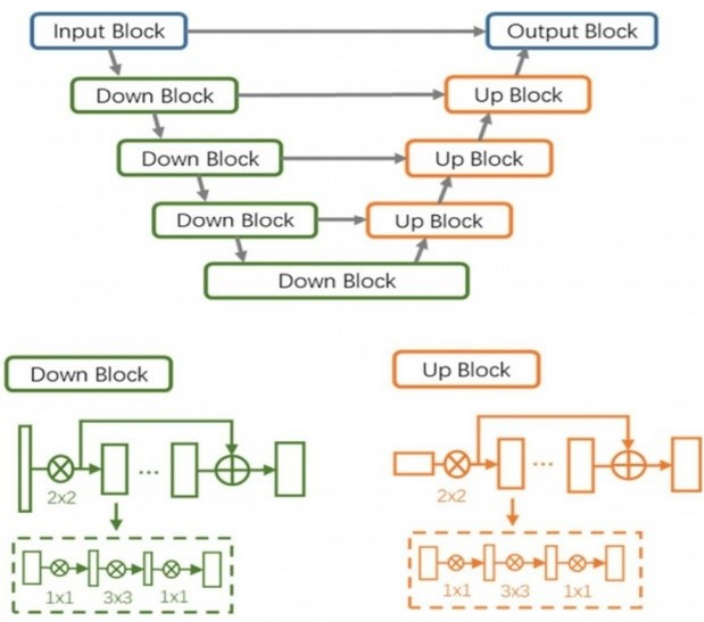

Figure 1 Network structure in [8] for COVID-19 infection segmentation.

In contrast, Figure 2 illustrating the proposed human-in-the-loop workflow strategy by the authors in [8]. Their DL-based segmentation has involved the "VB-Net" neural network to extract all COVID19 infection areas out of CT scan images. They have trained their system with 249 COVID- 19 cases, and validated it with other 300 new COVID-19 cases. We can see that they have also introduced a human-in-the-loop (HITL) strategy to be used during training phase of their model to speedup process of manual definition of CT scan images. This strategy has helped radiologists during the process of tagging some footnotes on each case's scanned image. The proposed model was implemented and tested across the conventional way of diagnosing the COVID19 to find that the proposed system could reduce 4 minutes after 3 iterations of model updating, in contrast with the fully manual delineation; which regularly may take time varied between 1 to 5 hours. In another attempt, Adrian Rosebrock in [14] has proposed a DL model for diagnoses COVID19 using Keras library and TensorFlow training platform.

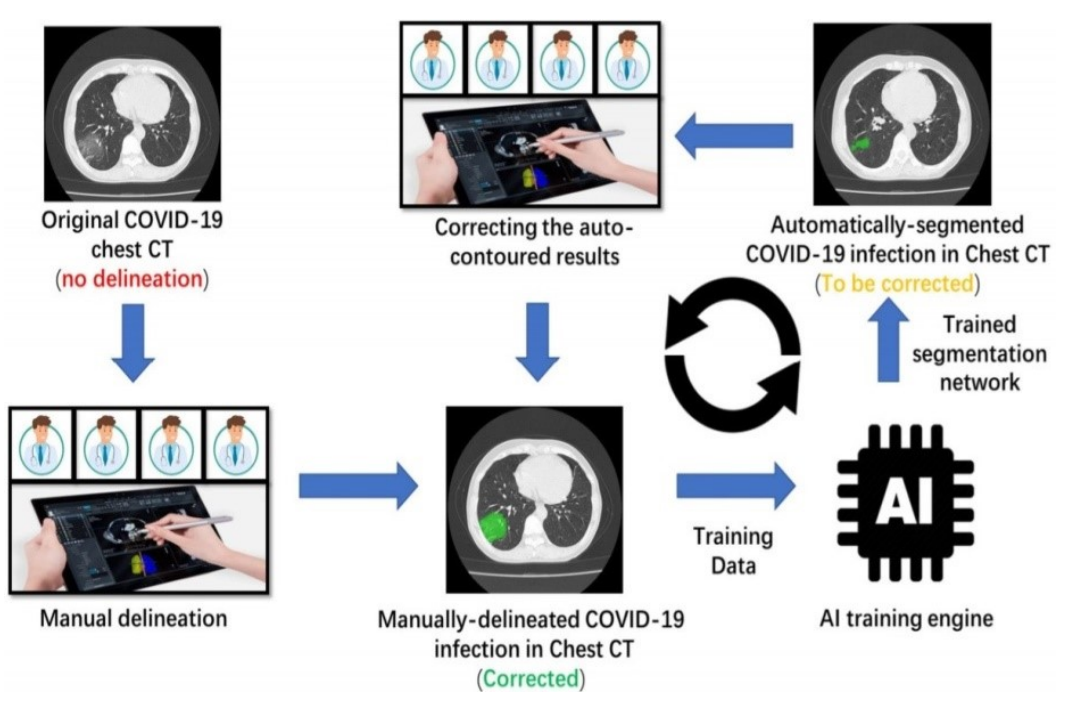

Figure 2 Human-in-the-loop workflow in [8]. 


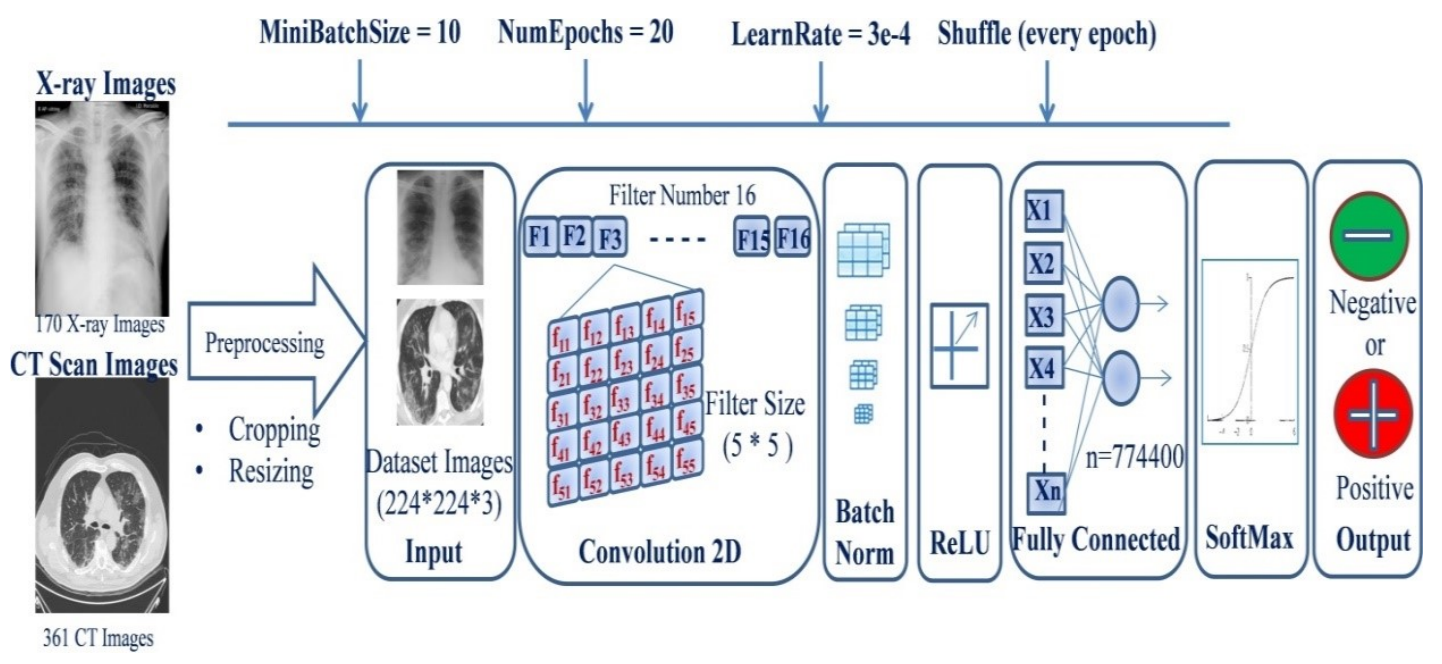

Figure 3 The modified CNN architecture to diagnose disease COVID-19.

Using in total 50 images, which has been equally divided into 25 positive COVID-19 and other 25 negative X-rays images; they have build, train and validate their model. Out of the conducted experiments, their proposed model could diagnoses COVID- 19 with average accuracy of $90-92 \%$, which was applied on their testing set with $100 \%$ sensitivity and $80 \%$ specificity due to the limited data. As been stated in the published work in [10], the proposed model still has high potential for improvement, as it was trained and tested only with relatively small dataset. Besides, the model has to be trained and learn numerous patterns, which are not highly related to COVID-19 so that can broad its diagnoses knowledge system. It is also crucial highlighting that having a relatively robust CONVID19 diagnoses system could be achieved via a multi-modal, which processes multi factors such as patient vitals, population density, geographical location and some others. Therefore, having a diagnoses system relying only on X-ray images will not be that reliable to ending such high risk epidemic of COVID-19 [14].

\section{THE PROPOSED APPROACH}

This section discusses the design of the proposed CNN approaches used to identify COVID-19 cases and details of proposed CNN model implementations. The proposal includes two main algorithms which a simple CNN architecture and a transfer learning algorithm, namely AlexNet. CNN algorithms are working by extracting relevant features through a series of convolutional layers followed by fully connected neural layers.

There are many types of deep learning algorithms ranging from convolution neural networks (CNN) to the recurrent neural networks ( $\mathrm{RNN})$. The $\mathrm{CNN}$ could be applied on these solutions when the data are retrieved in a spatial domain such as image processing applications [12]. But the RNN is working on the concept of re-using the output of each layer as an input for the next layers. Further, the RNN is compatible with those applications which are getting sequential data such text [15] and signal reading measurements [16]. While, the transfer learning is the concept of re-using a pre-trained network and transfer the learned model into a new model. The new models can also take new additional training data and modified neural layers [17]. In this study, for the purpose of diagnosing COVID-19 cases, a $\mathrm{CNN}$ architecture (as a deep learning algorithm) and modified AlexNet network (as a transfer learning algorithm) have been utilized. 


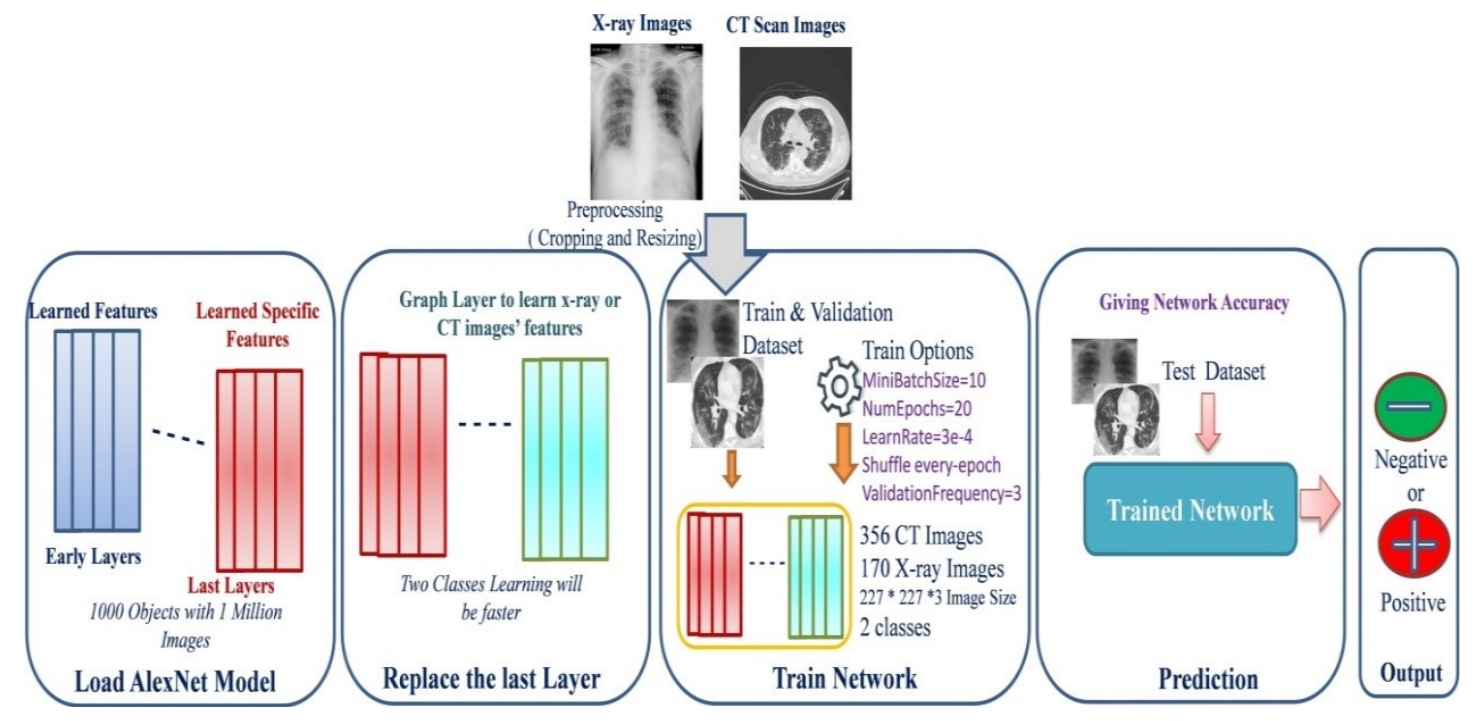

Figure 4 The modified AlexNet network for diagnosing disease COVID-19.

Proposed CNN architecture: To detect COVID-19 cases, we designed a very simple CNN model that consists of only one convolutional layer that constitute of 16 filters followed by batch normalization, rectified linear unit (ReLU), two fully connected layers, SoftMax and a classification layer. We adopted 'glorot' to initialize the weights and cross-entropy used as a loss function in the classification layer. Full details of our proposed CNN model is depicted in Figure 3.

Input layer: This layer is responsible to read a pre-processed image dataset. This means, a preprocessing step is applied on the X-ray and CT scan images, separately. The pre-processing step includes cropping and resizing the images. This is because, 1) when images are taken from medical devices, several letters, art craft and medical symbols are made on them, and 2) since the images come from different sources, their sizes will be varied. Therefore, in this study, the size of the input image is changed to 224-by-224-by-3 which is width-by-height-by-channel number. We cropped the lung and chest area such that it does not contain any writing, as much as possible, a sample of the images can be seen in fig 3 .

Convolution layer: This layer is the essential layer of our proposed CNN model in which most of the computations will be performed. The main function of this layer is to retrieve features from the image dataset and to maintain the spatial relationship between image pixels. This is obtained via learning the retrieved features using a set of filters. In this work, a two dimension convolution relied on 16 filters where each filter is constructed based on $5 * 5$ filter size. Further, the filters are moving along input images and calculating the dot product function which is known as convolved features. Practically, the $\mathrm{CNN}$ learns these convolved features during the training process and the convolved images are of the same size as the input image.

Batch Normalization Layer: This layer is a very deep neural network training technique that normalizes the convolved feature values. The main reason of using this layer is to reduce the number of training epochs which are required for training deep network and stabilizing the learning process. ReUL layer: The purpose of using such layer is replacing negative pixel values by zero in the convolved features. This is to produce the none-linearity map of the features in the CNN network.

Fully Connected Layer: The neurons at this layer are connected to all the activation functions from the previous layer. In this study, the main responsibility of this layer is to classify the retrieved convolved features from the image datasets into the defined classes. 
Table 1. Distribution of COVID-19 images (X-ray and CT) with respect to their collected sources.

\begin{tabular}{|c|c|c|c|}
\hline COVID-19 & GitHub & BSTI & Total \\
\hline X-ray & 70 & 15 & 85 \\
\hline CT & 16 & 187 & 203 \\
\hline
\end{tabular}

Softmax Layer: This layer is simply used to interpret the probabilities values of the activation function results from the previous layer. In the diagnose disease cases; the values could be interpreted into two classes which are ' 0 ' and ' 1 '.

Output Layer: This is the final layer of the CNN model in which the result values of the previous layer could be labeled. For example, the value of ' 1 ' is labeled to COVID-19 (i.e. positive case) and the value ' 0 ' is labeled to noneCOVID-19 (i.e. normal chest X-ray or CT).

Modified AlexNet Network: the pre-trained AlexNet network is referred to the CNN family which has been trained over a few million images on ImageNet in the range of one thousand different classes (or objects) [1]. The main purpose of using and modifying the pre-trained AlexNet is to transfer the learned weights, bias and features to the proposed approach (i.e. diagnosing disease COVID-19). This is followed by applying these parameters with training our new input dataset, i.e. CT scan images and $\mathrm{X}$-ray image dataset, separately. In addition, using such transfer learning algorithms provide insight into how one can think of design new architectures for the detection of COVID-19 cases. This is because; training a CNN network based on initializing random weights value from the scratch will take long time than tuning a pre-trained network and does not require a hug computational power especially if the dataset of interest does not contain a large number of images. However, to adopt the pre-trained network for our task, the model is modified based on replacing the last layers by intended layers. Further, our collected datasets have been used for further training network, as it can be seen in Figure 4.

The input images of the dataset are cropped and the size of the images are unified according to AlexNet model, when the width is equal to 227 , height is equal to 227 , and the channel color number is equal to 3 . Further, a set of parameters of the modified network are configured including minimum batch size is tuned to 10 , number of epochs is equal to 20 , learning rate is initialized to $3 \mathrm{e}-4$, shuffling is set at every epoch, and finally the validation frequency has been adjusted to 3 . The input images of the dataset are cropped and the size of the images are unified according to AlexNet model, when the width is equal to 227 , height is equal to 227 , and the channel color number is equal to 3 . Further, a set of parameters of the modified network are configured including minimum batch size is tuned to 10 , number of epochs is equal to 20 , learning rate is initialized to $3 e-4$, shuffling is set at every epoch, and finally the validation frequency has been adjusted to 3 .

\section{MATERIALS AND METHODS}

\section{A. Datasets}

To test the proposed approach, we collected images from 5 different sources to form a dataset of 170 X-ray images and 356 CT images of COVID-19 disease. The distribution of images according to their collected sources are list in Table 1 and Table 2. There are two reasons behind using the images from these sources. First, the diversity of the sources whereby the images collected from different repositories and countries. This is important to design a sophisticated tool to help radiologists to diagnose COVID-19 around the world. Second, the images from these sources are openly available to research community and to the general public. Furthermore, the images used in this work are collectively available in GitHub repository [18]. It is clear from Table 1 that we currently do not have plenty of COVID-19 images publicly available to the research community to conduct intense 
investigation.

Table 2: Distribution of Normal (No infection) chest images (X-ray and CT) with respect to their collected sources.

\begin{tabular}{|c|c|c|c|}
\hline Normal & Kaggle & Radiopedia & Total \\
\hline X-ray & 70 & 15 & 85 \\
\hline CT & - & 153 & 153 \\
\hline
\end{tabular}

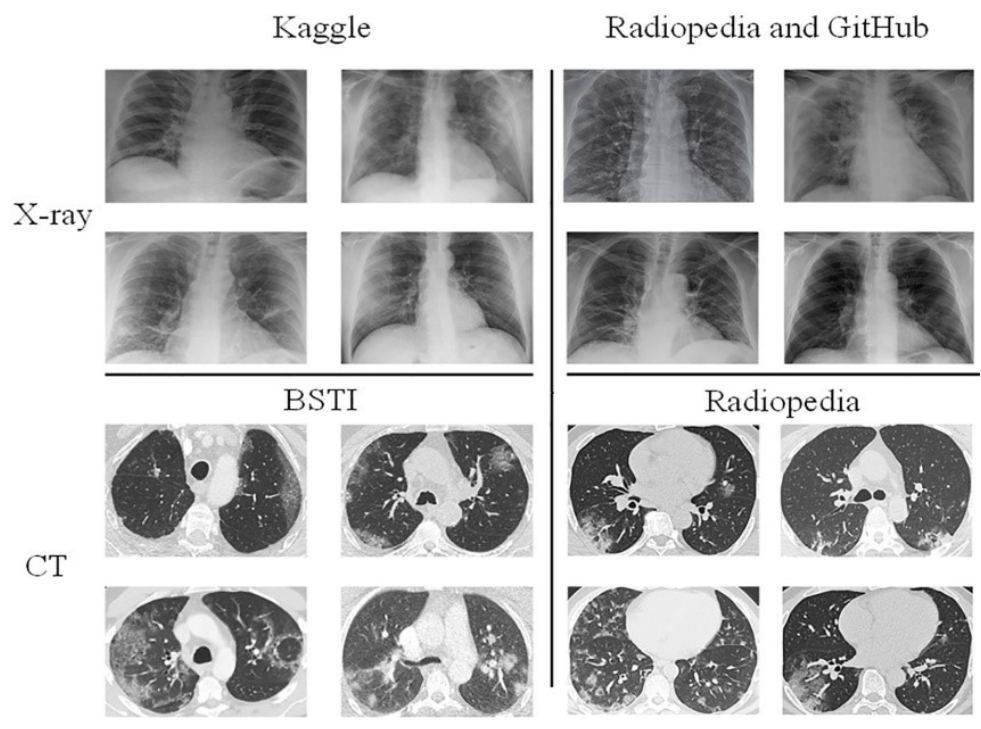

Figure 5 CT scan and X-ray images from different sources.

The X-ray images of COVID-19 are from 45 patients whereas the CT images are collected from 6 patients from British Society of Thoracic Imaging (BSTI) [19] dataset and 70 patients from GitHub, in which part of the images are collected from Societa Italiana di Radiologia Medica e Interventistica (SIRM) which stands for Italian Society of Medical Radiology and Interventional [20]. Figure 5 shows an example of the images that are used in our experiments. Both Datasets are updated regularly whereby hospitals around the world are uploading images of the disease. The images are collected from both of datasets until 18th March 2020. All COVID-19 images are confirmed cases, the patient ages are varying from 35 to 84 years old and of mixed gender.

The proposed CNN model is trained using $120 \mathrm{X}$-ray images (60 COVID19 from github, 60 normal from Kaggle [21]) and 339 CT images (COVID19: 187 BSTI and 5 from github, normal: 147 from Kaggle). More precisely, in the training phase the datasets are divided into two categories: $50 \%$ is used to train CNN whereas $50 \%$ is used to validate the model three times in each epoch (Figure 5).

To give credibility to the results, testing phase is adopted in the experiments. To test the proposed CNN models, we used 50 X-ray images (25 COVID-19 X-ray images from BSTI and 25 Normal X-ray images where we selected 10 images from Kaggle and 15 images from Radiopedia). For CT images, on the other hand, 17 images used in the testing phase whereby 11 of them is COVID-19 from GitHub and 6 normal CT images from radiopedia. Furthermore, it is worth mentioning that the performance measures of the experiments exhibit the average of 10 simulation runs (each run is 20 epochs with mini-batch of size 10). Note that the majority of images used in testing phase are from a different source which comes from a different device setting and a different country. The performance measures used in this study are accuracy (Acc), sensitivity and specificity which will be defined next. 
Table 3: Performance of proposes CNN and AlexNet for COVID-19 detection.

\begin{tabular}{|c|c|c|c|c|}
\hline Method & Image type & Sensitivity & Specificity & Acc \\
\hline \multirow{2}{*}{ Our CNN } & X-ray & 100 & 88 & 94 \\
\cline { 2 - 5 } & CT & 90 & 100 & 94.1 \\
\hline \multirow{2}{*}{ AlexNet } & X-ray & 100 & 96 & 98 \\
\cline { 2 - 5 } & CT & 72 & 100 & 82 \\
\hline
\end{tabular}

\section{Acc $=$ Truepositive $(T P)+\operatorname{Truenegative}(T N)$ \\ total number of testedimages}

where true positive is the number of truly identified COVID-19, true negative is the number of truly identified non-COVID-19 (normal) cases.

$$
\text { sensitivity }=\frac{T P}{T P+F a l \text { senegaitue }(F N)}
$$

where FN is the number of COVID-19 images (X-ray or CT) incorrectly classified as non-COVID-19 and FP is the number of normal (X-ray or CT) images misclassified as COVID-19.

$$
\text { specificity }=\frac{T N}{T N+\text { Falsepositive }(F P)}
$$

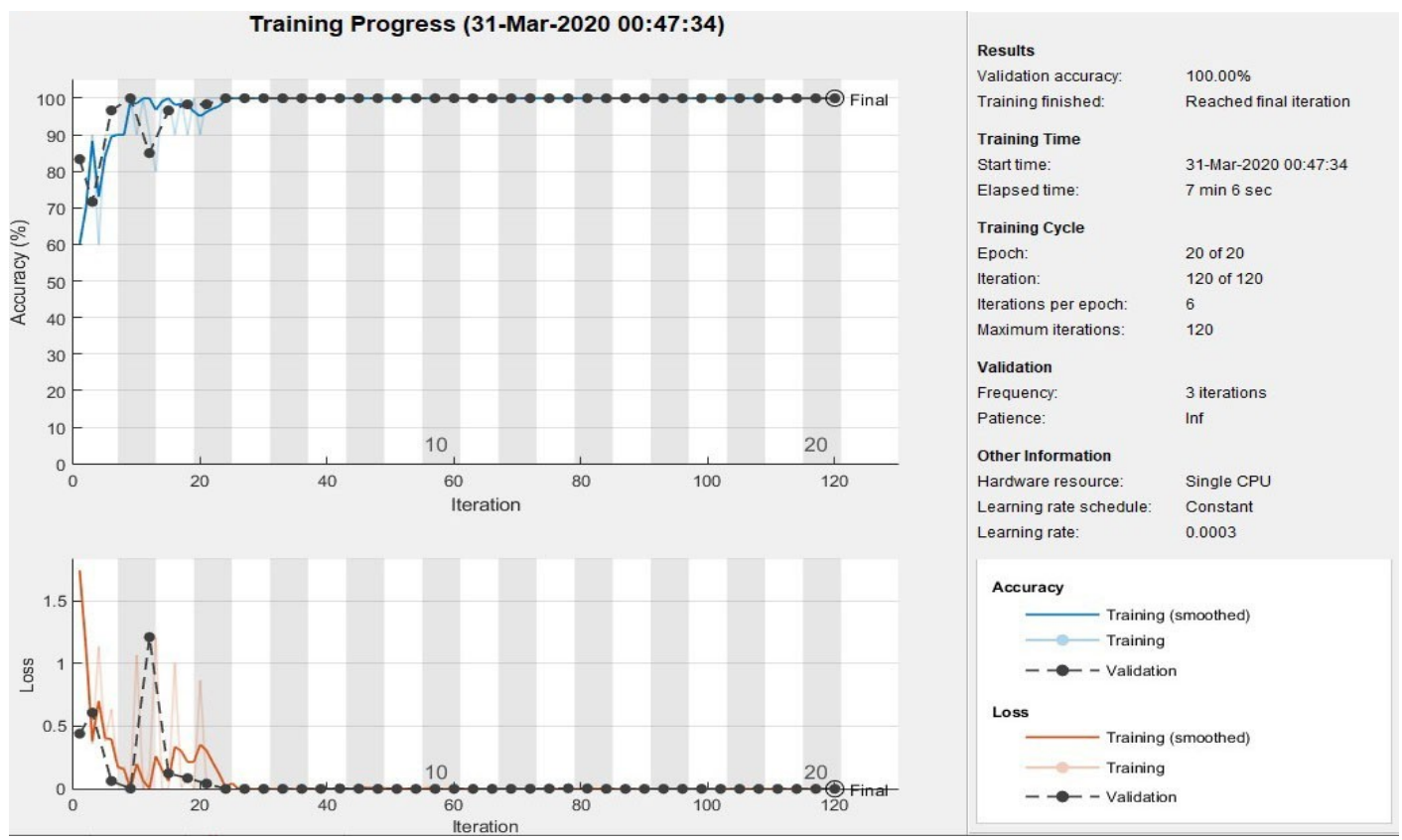

Figure 6 Validation accuracy with respect to the 120 iterations.

\section{B. Experiments \& Results}

This section presents the results of the proposed CNN architecture and it is listed in Table 3. It is clear from Table 3, the proposed CNN model performs well on both types of images used in our experiments, which are $\mathrm{X}$-ray and $\mathrm{CT}$. 


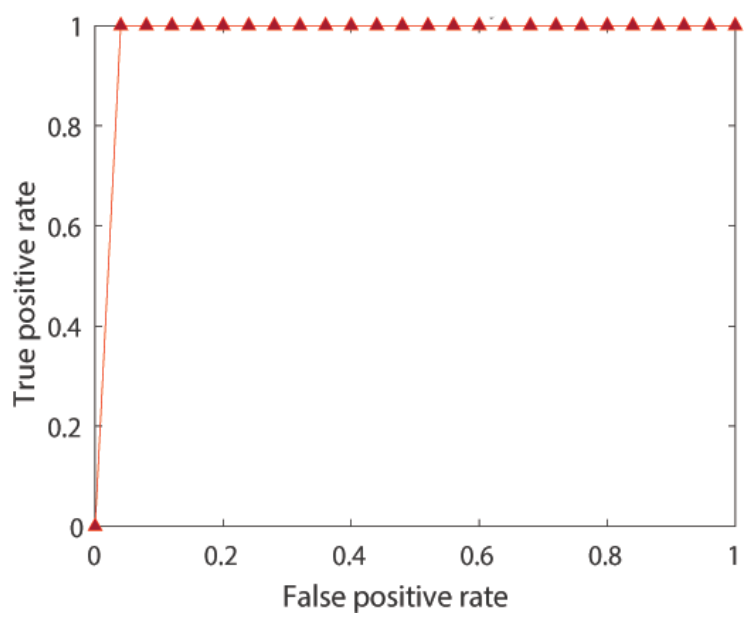

Figure 7 True positive rate with respect to the false positive rate.

Interestingly, the proposed model achieves $100 \%$ sensitivity when X-ray is used to test the suspected patients for COVID-19 and $90 \%$ sensitivity achieved when CT images are used. Pretrained AlexNet, on the other hand, is not performing bad on X-ray images whereby it correctly identified all COVID-19 images and correctly identified $96 \%$ of the normal X-ray images. But this is not the case when pretrained AlexNet used to differentiate COVID-19 CT images from their normal counterparts such that it only classified $72 \%$ of the COVID-19 CT images correctly. In comparison with a newly published CNN architecture in [1], where the same dataset from GitHub and Kaggle used, the CNN method in [1] achieved the same sensitivity as ours on COVID-19 X-ray chest images while our proposed CNN sensitivity is better than [1] for Normal X-ray images. Furthermore, there is no hint whether the CNN method proposed in [1] will work on CT images or not, whereas the CNN proposed in this study shows promising results to be used to detect COVID-19 despite the small number of images used in the current investigation. Finally, it should be noted that the CNN model proposed in this work is a very simple, yet effective, architecture which is main pillar is of only one convolutional layer which constitutes of 16 filters of size 5-by-5 trained from scratch using the weight glorot wrightinitializer, see Figure 1.

Pretrained AlexNet, on the other hand, performed well to differentiate COVID-19 X-ray images from normal X-rays, but not as good as X-ray scans of chest radiographs when it was tested on CT images of COVID-19 patients. Other performance metrics of Pretrained AlexNet on COVID-19 and normal Xrays are illustrated by Figure 6 and 7. As it is shown in Figure 6, the validation accuracy is $100 \%$ in 20 epoch. This is due to the fact that the Pretrained AlexNet is modified by replacing last layers with intended layers. Further, training the Pretrained AlexNet is not adopted from scratch as conventional CNN. Pretrained AlexNet transfer learning algorithm exhibits a great performance on extracting silent features of the COVID-19 X-ray images (Figure 8). As depicted in Figure 8, the improved AlexNet diagnoses all COVID-19 X-ray images whereas it could correctly identified 23 normal X-ray images out of 25 images, this means the overall accuracy is around $98 \%$. The results show the efficiency of AlexNet in detection of lesions and opacities of infected COVID-19 patients. This will tremendously assist the radiologists by overcoming load on the medical system and hospitals.

\section{CONCLUSION}

In this study, we introduced a simple yet an effective CNN model together with testing pre-trained AlexNet for the detection of COVID-19 disease from chest X-ray and CT images that is available 
publicly. Furthermore, the images (X-ray and CT) used in our investigation are collected from multiple sources and we collectively make the radiography images used in this study to detect COVID-19 will be publicly available for the research community.

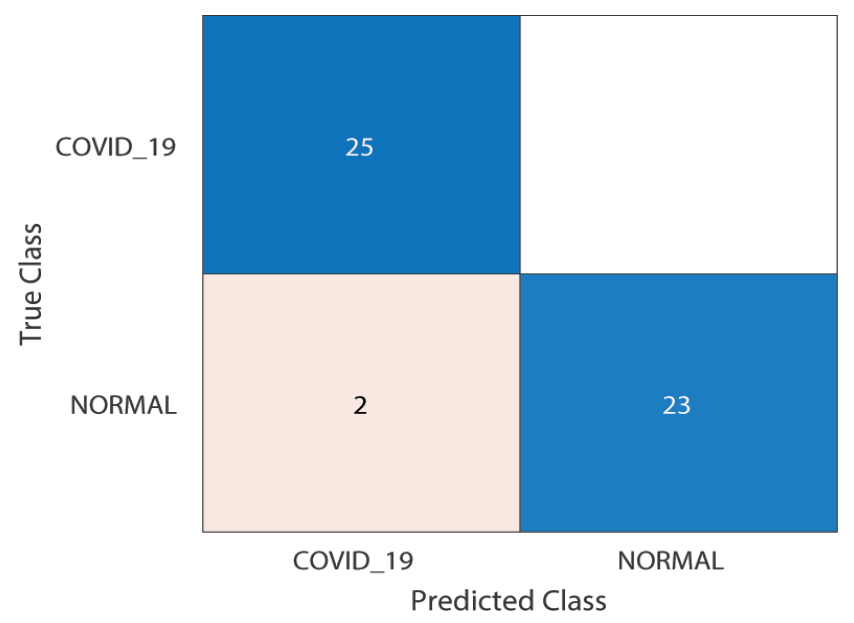

Figure 8 Identified all COVID-19 X-ray images correctly, while 23 normal X-rays images out of 25 images.

Although we achieved rather high COVID-19 detection accuracy, sensitivity and specificity but this does not mean a production ready solution especially with the limited number of images currently available about COVID-19 cases. The purpose of this study is to provide radiologists, data scientists and research community with a simple CNN model which can be adopted for early diagnosis of COVID-19 and hopefully this will be built upon to accelerate the research in this direction. Also, further efforts are required to develop a system to deploy and run the proposed models on current powerful technologies, such as smartphones and Tablets. This is to help the radiologist to use the system which can be deployed on cell phones especially nowadays majority of people have smartphones with good computational power. Hence, if one can build a simple CNN model and its effective then it is easy, and does not require a lot of computational power, for testing prospective radiography images and can also be adapted easily on smartphones too. In future, we plan to collect more X-ray and CT images to expand the investigation presented in this study and if needed design a deeper CNN accordingly. We hope that the results presented in this study serves as a small step towards building a sophisticated COVID-19 disease detection from X-ray or Ct images sooner than later to save as many live as possible. Although the accuracy of the proposed models is not enough, but the result of the detection could be compensated by using other symptoms of the disease. The symptoms' level could be clearly identified via reading sensing data of onboard smartphones sensors including fever symptom level via temperature sensor, or fatigue symptom via inertial sensor, or cough symptom via microphone sensor [22].

\section{REFERENCES}

[1]J. Chen, L. Wu, J. Zhang, L. Zhang, D. Gong, Y. Zhao, S. Hu, Y. Wang, X. Hu, B. Zheng et al., "Deep learning-based model for detecting 2019 novel coronavirus pneumonia on high-resolution computed tomography: a prospective study," medRxiv, 2020.

[2]M. Holshue, C. DeBolt, and L. S. First, "Novel coronavirus in the united states," N Engl J Med, vol. 2020, p. 31, 2019.

[3]"Novel coronavirus - china. world health organization." https://www. who.int/csr/don/12-january- 
2020-novel-coronavirus-china/en/., Accessed in March, 2020.

[4]"Cnn health," https://edition.cnn.com/2020/03/11/health/coronavirus-pandemic-world-healthorganization/index.html, Ac- cessed in March, 2020.

[5]D. Wang, B. Hu, C. Hu, F. Zhu, X. Liu, J. Zhang, B. Wang, H. Xiang, Z. Cheng, Y. Xiong et al., "Clinical characteristics of 138 hospitalized patients with 2019 novel coronavirus-infected pneumonia in wuhan, china," Jama, 2020.

[6] National health commission of china. ( March, 2020). [Online]. Available: http://www.chinadaily.com.cn/m/chinahealth/index.html

[7] Canada broadcast cooperation. (2020, March). [Online]. Available: https://www.cbc.ca/player/play/1709650499517

[8]F. Shan+, Y. Gao+, J. Wang, W. Shi, N. Shi, M. Han, Z. Xue, D. Shen, and Y. Shi, "Lung infection quantification of covid-19 in ct images with deep learning," arXiv preprint arXiv:2003.04655, 2020.

[9]Z. Y. Zu, M. D. Jiang, P. P. Xu, W. Chen, Q. Q. Ni, G. M. Lu, and L. J. Zhang, "Coronavirus disease 2019 (covid-19): A perspective from china," Radiology, p. 200490, 2020.

[10]C. Huang, Y. Wang, X. Li, L. Ren, J. Zhao, Y. Hu, L. Zhang, G. Fan, J. Xu, X. Gu et al., "Clinical features of patients infected with 2019 novel coronavirus in wuhan, china," The Lancet, vol. 395, no. 10223, pp. 497-506, 2020.

[11]N. Chen, M. Zhou, X. Dong, J. Qu, F. Gong, Y. Han, Y. Qiu, J. Wang, Y. Liu, Y. Wei et al., "Epidemiological and clinical characteristics of 99 cases of 2019 novel coronavirus pneumonia in wuhan, china: a descriptive study," The Lancet, vol. 395, no. 10223, pp. 507-513, 2020.

[12]F. Milletari, N. Navab, and S.-A. Ahmadi, "V-net: Fully convolutional neural networks for volumetric medical image segmentation," in 2016 Fourth International Conference on 3D Vision (3DV). IEEE, 2016, pp. 565-571.

[13]K. He, X. Zhang, S. Ren, and J. Sun, "Deep residual learning for image recognition," in Proceedings of the IEEE conference on computer vision and pattern recognition, 2016, pp. 770-778.

[14]B. P. Roquette, H. Nagano, E. C. Marujo, and A. C. Maiorano, "Prediction of admission in pediatric emergency department with deep neural networks and triage textual data," Neural Networks, 2020.

[15]M. I. Razzak, S. Naz, and A. Zaib, "Deep learning for medical image processing: Overview, challenges and the future," in

Classification in BioApps. Springer, 2018, pp. 323-350.

[16]C. Lin, Y. Ding, B. Xie, Z. Sun, X. Li, Z. Chen, and M. Niu, "Asymptomatic novel coronavirus pneumonia patient outside wuhan: The value of ct images in the course of the disease," Clinical Imaging, 2020.

[17]S. Rajaraman, S. K. Antani, M. Poostchi, K. Silamut, M. A. Hossain, R. J. Maude, S. Jaeger, and G. R. Thoma, "Pre-trained convolutional neural networks as feature extractors toward improved malaria parasite detection in thin blood smear images," PeerJ, vol. 6, p. e4568, 2018.

[18]J. P. Cohen, P. Morrison, and L. Dao, "image data collection," arXiv 2003.11597, vol. 6, 2020.

[19]Covid-19 bsti imaging database. (2020, March). [Online]. Available: https://www.bsti.org.uk/training-and-education/ covid-19-bsti-imaging-database/

[20]C. Hacking and I. Bickle. Normal chest imaging examples. (2020, March). [Online]. Available: Radiopaedia,https:

//radiopaedia.org/articles/normal-chest-imaging-examples?lang=gb

[21]P. Mooney. Chest x-ray images (pneumonia). ( March, 2020). [Online]. Available: https://www.kaggle.com/ paultimothymooney/chest-xray-pneumonia/metadata

[22]H. S. Maghdid, K. Z. Ghafoor, A. S. Sadiq, K. Curran, and K. Rabie, "A novel ai-enabled framework to diagnose coronavirus covid 19 using smartphone embedded sensors: Design study," arXiv preprint arXiv:2003.07434, 2020. 